\title{
Intracerebral propagation of interictal activity in partial epilepsy: implications for source localisation
}

\author{
G Alarcon, C N Guy, C D Binnie, S R Walker, R D C Elwes, C E Polkey
}

\section{Abstract}

The hypothesis that focal scalp EEG and MEG interictal epileptiform activity can be modelled by single dipoles or by a limited number of dipoles was examined. The time course and spatial distribution of interictal activity recorded simultaneously by surface electrodes and by electrodes next to mesial temporal structures in 12 patients being assessed for epilepsy surgery have been studied to estimate the degree of confinement of neural activity present during interictal paroxysms, and the degree to which volume conduction and neural propagation take part in the diffusion of interictal activity. Also, intrapatient topographical correlations of ictal onset zone and deep interictal activity have been studied. Correlations between the amplitudes of deep and surface recordings, together with previous reports on the amplitude of scalp signals produced by artificially implanted dipoles suggest that the ratio of deep to surface activity recorded during interictal epileptiform activity on the scalp is around 1:2000. This implies that most such activity recorded on the scalp does not arise from volume conduction from deep structures but is generated in the underlying neocortex. Also, time delays of up to $220 \mathrm{~ms}$ recorded between interictal paroxysms at different recording sites show that interictal epileptiform activity can propagate neuronally within several milliseconds to relatively remote cortex. Large areas of archicortex and neocortex can then be simultaneously or sequentially active via three possible mechanisms: (1) by fast association fibres directly, (2) by fast association fibres that trigger local phenomena which in turn give rise to sharp/slow waves or spikes, and (3) propagation along the neocortex. The low ratio of deep-to-surface signal on the scalp and the simultaneous activation of large neocortical areas can yield spurious equivalent dipoles localised in deeper structures. Frequent interictal spike activities can also take place independently in areas other than the ictal onset zone and their interictal propagation to the surface is independent of their capacity to trigger seizures. It is concluded that: (1) the deep-to-surface ratios of electromagnetic fields from deep sources are extremely low on the scalp; (2) single dipoles or a limited number of dipoles are not adequate models for interictal activity for surgical assessment; (3) the correct localisation of the onset of interictal activity does not necessarily imply the onset of seizures in the region or in the same hemisphere. It is suggested that, until volume conduction and neurophysiological propagation can be distinguished, semiempirical correlations between symptomatology, surgical outcome, and detailed presurgical modelling of the neocortical projection patterns by combined MEG, EEG, and MRI could be more fruitful than source localisation with unrealistic source models.

$(\Im$ Neurol Neurosurg Psychiatry 1994;57:435-449)

A successful outcome of resective surgery for epilepsy depends on the localisation of an epileptogenic zone in nervous tissue that is structurally and functionally abnormal. Recent developments in medical imaging have provided a substantial improvement in the localisation of structural lesions. The need for identification of functional abnormalities, however, still demands recording the onset of ictal activity in a high proportion of patients assessed for surgery for epilepsy. In particular, deep temporal and frontal structures are often involved in the genesis of epileptic seizures and the localisation of the ictal onset zone may require the chronic implantation of intracranial electrodes. Presently, attention to the onset and propagation of ictal events is usually emphasised during the interpretation of intracranial recordings (for reviews, see Wieser $^{1}$ and Spencer ${ }^{2}$ ). Interictal paroxysmal events (spikes and sharp waves) are, however, recorded from both deep and superficial structures. These can help the understanding of neurophysiological mechanisms involved in the generation of scalp epileptiform activity and the clinical interpretation of scalp electroencephalograms. The analysis of scalp interictal activity has been suggested as an alternative to intracranial recordings, largely based on the assumption that synchronised neural activity remains relatively localised during interictal paroxysms. Accordingly, the electric and magnetic fields generated on or around the scalp by interictal epileptic activity have been analysed to localise epileptogenic foci. ${ }^{3-7}$ The models often assume that cellular currents produced by synchronised neuronal 
activity (primary or impressed currents) generate an electromagnetic field, which in turn induces secondary currents in the surrounding conducting medium (volume currents). In principle, both impressed and volume currents can contribute to the amplitude of the scalp electric and magnetic fields (electroencephalogram (EEG) and magnetoencephalogram, (MEG)). Several algorithms have been developed to localise the impressed currents from scalp EEG or MEG measurements. Most methods model the impressed current as a current dipole or a restricted number of dipoles. ${ }^{8-10}$

Although source localisation by means of scalp EEG modelling has now been used for over 15 years, it has never become a standard clinical tool. It is presumed that one of the reasons is that volume currents resulting from inhomogeneities in medium conductivity or differences between the assumed geometrical head model and the real head shape can smear or displace source localities. It can be demonstrated that, in a spherical conductor, the component of the external magnetic field normal to the surface does not contain contributions from volume currents. ${ }^{11}$ This has motivated several investigators to evaluate interictal $M E G$ recordings as a non-invasive alternative to deep recording for localising epileptogenic foci during preoperative assessment. Various publications have claimed that scalp MEG can be successfully used for this purpose. ${ }^{34612}$ Nevertheless, Cohen et al found that EEG and MEG achieved similar accuracy in localising current dipoles generated by intracranial electrodes implanted in patients. ${ }^{13}$ This finding has triggered a lively discussion among MEG researchers. ${ }^{14}$

Surprisingly, although theoretical physical models of interictal activity have been thoroughly discussed, the neurophysiological mechanisms involved in interictal phenomena in humans and their correlations with ictal events are little understood. Moreover, as well as the potential relevance of scalp EEG and MEG activities in non-invasive surgical assessment, a neurophysiological understanding of interictal activity would be highly useful to the clinician, as most electroencephalographic studies carried out on patients with epilepsy are based on interictal recordings.

In the present paper we have analysed human interictal activity recorded simultaneously by surface electrodes and deep electrodes situated in or near mesial temporal structures and sometimes in the frontal lobe. Three main topics are considered: (1) the degree of confinement of neural activity during interictal paroxysms, (2) the degree to which instantaneous volume conduction (electromagnetic propagation) and neural conduction take part in the propagation of interictal activity, and (3) the topographical relation between interictal and early ictal activity. We discuss the complexities associated with interictal events, their influence in the localising capabilities of scalp recordings, and comment on the most appropriate mathematical models for the source.

\section{Methods}

Intracranial and scalp EEG recordings of interictal events from 12 patients under telemetric presurgical assessment for epilepsy surgery have been studied. Patient ages were between 22 and 45 (mean 29.7) years. Antiepileptic medication was reduced or withdrawn if seizures were not recorded by the second or third day of telemetry.

\section{PATIENT SELECTION}

All patients had partial epilepsy refractory to medical treatment, had complex partial seizures, and were not considered candidates for hemispherectomy, callosotomy or for "lesionectomy" (on grounds of lack of a discrete lesion on neuroimaging at a site concordant with clinical, interictal EEG, and neuropsychological findings). At our centre, $74 \%$ of patients referred for epilepsy surgery fall in this category and undergo telemetry with intracranial foramen ovale (FO) electrodes. Failure to demonstrate a probable temporal ictal onset zone or non-convergence between different sources of evidence concerning the likely source of seizures leads to further investigation by stereoelectroencephalography (SEEG). For a more detailed account of considerations that presently lead to selection for intracranial recordings, see Binnie et al. ${ }^{15}$

\section{RECORDING PROTOCOLS \\ Patients with scalp and FO electrodes (six patients)}

A flexible bundle of six electrodes was inserted bilaterally through the FO under fluoroscopic control. ${ }^{16}$ Each electrode consisted of a $0.1 \mathrm{~mm}$ stainless steel isolated wire with a $5 \mathrm{~mm}$ recording contact. Contacts from contiguous electrodes were located 10 $\mathrm{mm}$ apart along the bundle. Electrodes in the same bundle are labelled from 1 (the most superficial contact) to 6 (the deepest contact). Postinsertion radiography was performed to confirm that the FO electrode was lying between the mesial aspect of the temporal lobe and the brainstem. A full set of standard EEG electrodes was attached to the scalp according to the Maudsley system, which provides a more comprehensive coverage of the temporal regions than does the standard international $10 / 20$ system. ${ }^{17}$ Telemetry, which included simultaneous FO and scalp recordings, was started less than 36 hours after FO electrode implantation and continued for one to two weeks.

\section{Patients with subdural and intracerebral electrodes (six patients)}

A frontocentral trephine was performed to implant subdural seven-electrode bundles along the orbitofrontal, laterofrontal, anterior temporal, posterior temporal, and parietal surfaces of both cerebral hemispheres. Each subdural electrode consisted of a $0.1 \mathrm{~mm}$ stainless steel isolated cable. Contacts of the three deepest contiguous electrodes (labelled 7,6 , or 5 ) were $2.5 \mathrm{~mm}$ long and their centres were located $5 \mathrm{~mm}$ apart along the bundle. 
Contacts from the four more superficial electrodes (labelled $4,3,2$, or 1 ) were $5 \mathrm{~mm}$ long and their centres were situated $10 \mathrm{~mm}$ apart along the bundle. Intracerebral electrodes of similar characteristics were bilaterally implanted under CT control in the amygdala, anterior and posterior hippocampus, and occasionally in the medial frontal region. ${ }^{18}$ Telemetric recording from intracerebral and subdural electrodes commenced three to seven days after implantation and continued for up to three weeks. Scalp EEG was recorded in one patient simultaneously with intracerebral and subdural recordings to estimate the signal attenuation produced by the skull and scalp.

Although FO electrodes are subdural electrodes in the sense that they are immersed in the subdural space, they supposedly record from deep brain structures (mesial temporal structures). Therefore we grouped FO and intracerebral electrodes as "deep electrodes" to simplify terminology in this paper. We will generically designate scalp electrodes in patients with FO insertions and subdural electrodes in patients with intracerebral electrodes as "surface electrodes", as they lie over the convexity of the neocortex.

RECORDING SYSTEM AND ANALYSIS

Cable telemetry of 32 or 64 channels was used for data acquisition (Telefactor Beekeeper system). Data were digitalised at $200 \mathrm{~Hz}$ and band filtered (high pass cutoff frequency at $0.3 \mathrm{~Hz}$ and low pass cutoff frequency at $70 \mathrm{~Hz}$ ). The system input range was $2 \mathrm{mV}$ and data were digitalised with a $12 \mathrm{bit}$ analog-to-digital converter (an amplitude resolution of $0.488 \mu \mathrm{V}$ ). All data were recorded as common reference. $\mathrm{Pz}$ was used as common reference during display of data from patients with FO electrodes. For data display from intracerebral electrodes the selection of the reference varied, but was usually a remote scalp reference. Sections of 10-20 minutes of interictal recordings from each patient were transcribed on to hard disk and paper. The topography and waveform of interictal events occurring within the transcribed sections were studied visually (a total of 651 events) and 45 spikes and sharp waves were selected for computer analysis. Although most patients showed more than one type of interictal event regarding waveform and spatial distribution, all events selected for computer analysis fulfilled two criteria: (1) they appeared as sharp spikes or spikes in at least one of the recording sites (events consisting solely of a paroxysmal burst of slow activity, with no spike component in any recording channel, were discarded). (2) Their waveform and topography occurred relatively frequently and consistently within each patient.

Selected interictal events were saved on floppy disks and data analysis was carried out on an IBM compatible personal computer with software developed in Assembler and QuickBASIC. Specific channels were expanded in time and amplitude (figs $1 \mathrm{C}$ and
1D). Cursors running through the data were used to measure latency and amplitude differences between channels. Because interictal events recorded by depth electrodes can show enormous variability in amplitude, polarity, and waveform within and between patients, no general mathematical technique for signal processing was applied to all selected events. Spectral analysis, cross correlation, complex demodulation, and digital filtering were used as appropriate to display relations among channels. Statistical dispersion is given as $\mathrm{SD}$ in all cases. Approximate surface amplitudes produced by dipole currents located in mesial temporal structures have been estimated for an unbounded homogeneous medium (formulae 12 and 13 from Sarvas ${ }^{11}$ ), with conductivities for grey and white matter given by Barber and Brown. ${ }^{19}$

\section{Results}

TYPES OF INTERICTAL ACTIVITY ACCORDING TO SPATIOTEMPORAL DISTRIBUTION

Consistent patterns of interictal activity were classified according to the morphology and spatiotemporal distribution of interictal events across deep and surface recordings. Five distinct types of interictal activity emerged. More than one type was usually present in any one patient.

Type I: events recorded only by deep electrodes (deep only, "DO", figs 2 and 3)

Type I was interictal activity recorded by deep electrodes but not identifiable as epileptiform activity in surface recordings except by referring to simultaneous deep recordings (see figs 2 and 3 ). This type was observed in 11 patients and was the most frequent type of interictal activity in eight patients.

Type II: events recorded in depth and surface with no latency differences (synchronous, "Synch", figs 2 and 4)

This type (present in 11 patients) corresponds to interictal spikes that showed latency differences shorter than $10 \mathrm{~ms}$ (two samples) between depth and scalp recordings or between different depth or scalp electrodes. These recordings often appear as synchronous events in surface and depth. All channels that record this type of event show similar waveforms although with different amplitude or polarity (fig 4 ).

Type III: events that showed latency differences of 10-50 ms between different recording sites (short latency, "SL", fig 3)

In this type, interictal events recorded by depth and surface electrodes may appear as synchronous spikes at the standard EEG paper speed. On an expanded time scale, latency differences can be seen of up to $50 \mathrm{~ms}$ between the beginning or between the peaks of interictal events in depth and surface recordings, or between different depth or surface electrodes. This type was present in nine patients. 
Figure 1 Propagation of interictal activity along the mesial aspect of the right temporal lobe in patient 7 (A) and (B) Scalp and $F O$ recordings of two examples recorded in the same patient. (C) and (D) Same events on expanded time scale. The events increase in amplitude and in duration as they propagate, presumably recruiting more neuronal activity for $a$ longer time. On the scalp the signal is recorded with largest amplitude at F8 but also at $F p 2, A 2$, and T4. The spike at RFO2 takes place $40 \mathrm{~ms}$ after the initial spike at RFO3. Note the striking similarities

between recordings at $\mathrm{F8}$

and RFO1. Both electrodes record mainly a high

amplitude sharp wave in

the alpha range $(9 \mathrm{~Hz})$

with a $105 \mathrm{~ms}$ delay with respect to the initial $R F O 3$ spike. Electrodes RFO1

and $F 8$ are about $4 \mathrm{~cm}$

apart and show nearly the

same trace, regardless of

different amplitudes

probably due to bone

attenuation. On the

contrary, RFO2 and

$R F O 3$ are 1 and $2 \mathrm{~cm}$

apart from $\mathrm{FO} 1$ and all

three show different

waveforms. Note that the

main deflections on RFO2,

$R F O 1$, and $F 8$ are

preceded by small.

amplitude deflections.

RFO2 spike is

preceded by a low

amplitude rhythm that

starts simultaneously with

RFO3 spike. RFO1 and

scalp main deflections are

preceded by a low

amplitude notch that takes

place 15-20 ms after

RFO3 spike. Numbers to

the right of traces indicate

the relative display gain for

the corresponding channels

and the ones below.

Calibration marks

correspond to $400 \mu \mathrm{V}$ in $\mathrm{A}$ and $B$, and $200 \mu V$ in $C$

and $D$, in the channels

with relative gain of 1 .
A

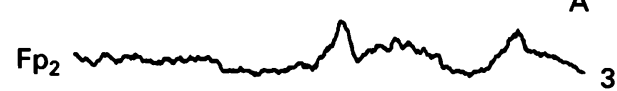

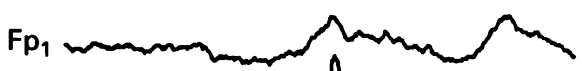<smiles>CCCCCCCC1CC1CCCCCCCCF</smiles>

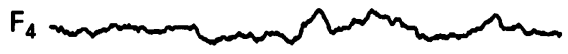

$F_{2}$

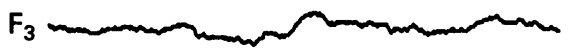

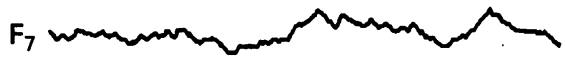

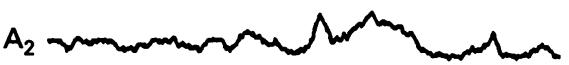

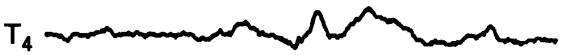

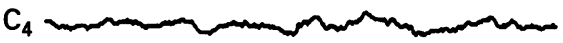

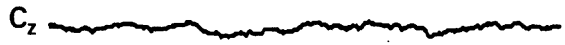

$\mathrm{C}_{3}$

$T_{3}$

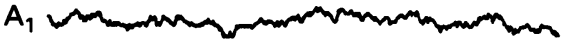
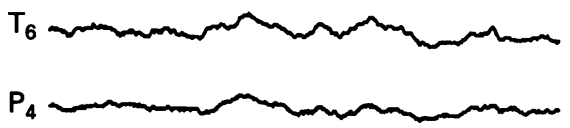

$P_{3}$
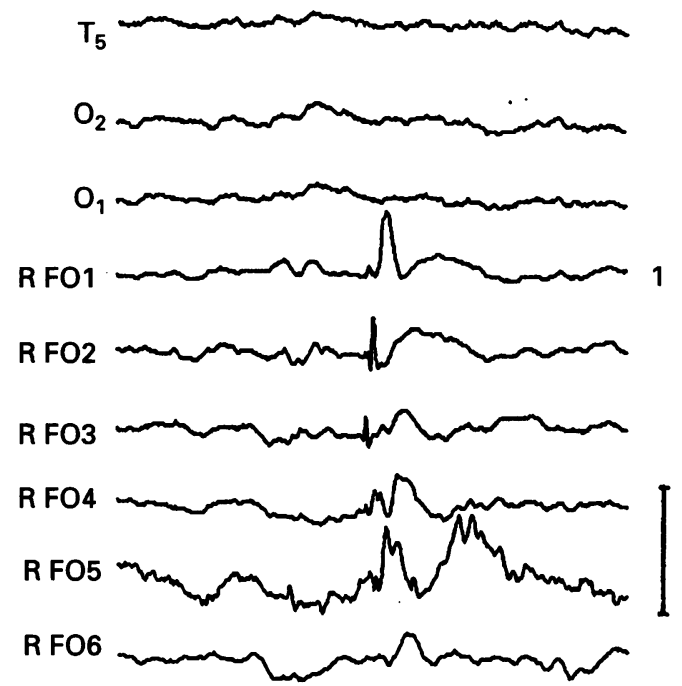

LFO3

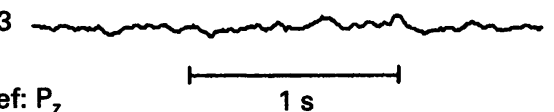

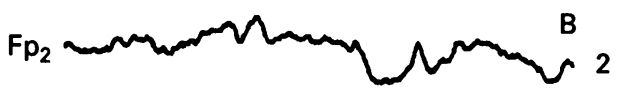

$\mathrm{Fp}_{1}$

$F_{8}$ mang Noming

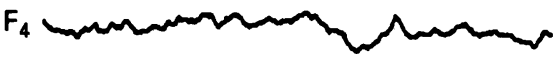

r

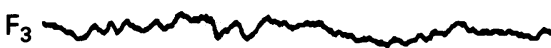

${ }_{7}$ Ormoms

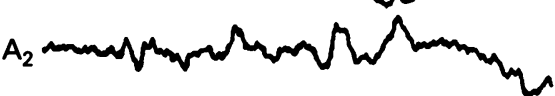
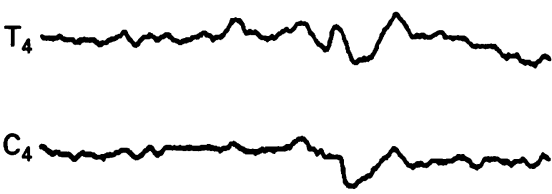

$\mathrm{C}_{2}$
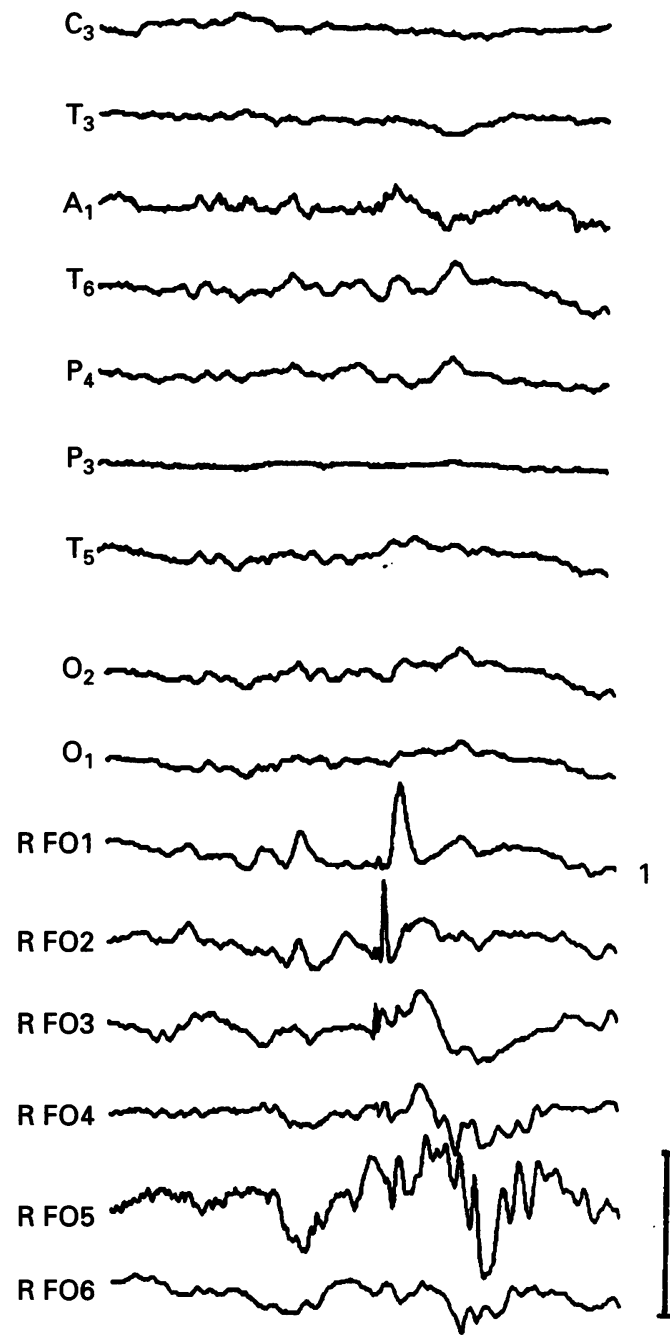

L FO3

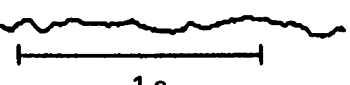

Ref: $P_{z}$ 


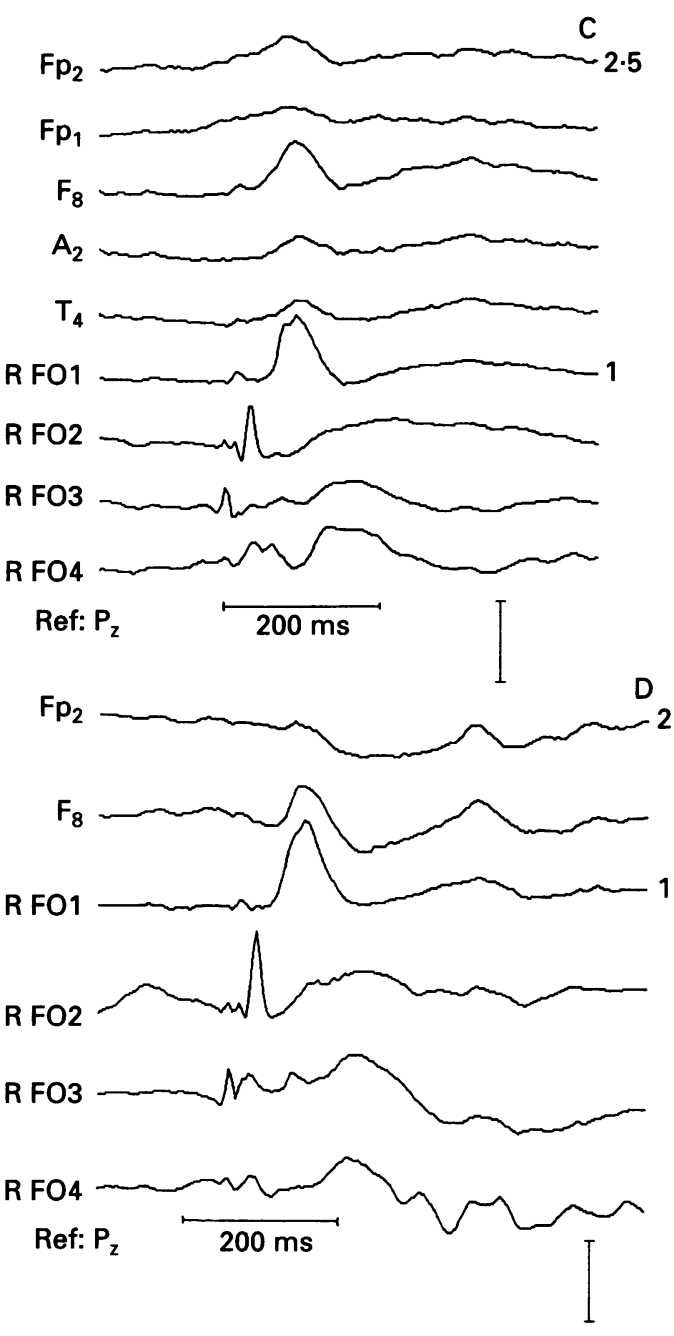

Type IV: events which showed latency differences larger than $50 \mathrm{~ms}$ between different recording sites (long latency, " $L L$ ", figs $1,3 B, 5$, and 6)

Six patients showed latency differences of 50 to $220 \mathrm{~ms}$ between deep and surface recordings, or between different deep or surface electrodes. In this type of event, deflections were recorded by some depth and some surface electrodes, regardless of their latency differences. The same range of latency differences was seen whether the beginning or the peak of the transient waveform was considered. Often in this type of interictal activity, the spike component in the depth had already ended when a sharp or slow wave was recorded on the surface, and the waveform was often different between surface and depth. Therefore, only consistent multichannel patterns that were repeatedly recorded in the same patient were considered, to confirm that the large latency differences did not represent recordings from independent asynchronous interictal foci. About $70 \%$ of events corresponding to this type of interictal activity were preceded by some form of low amplitude deflection in the surface EEG. These included: (1) low amplitude (10-40 $\mu \mathrm{V}$ ) deflections, synchronous with a spike in the depth; (2) low amplitude deflections $(10-40 \mu \mathrm{V})$ with delays (SL or LL) with respect to deep spikes (figs 1 and 6); or (3)
$\mathrm{FP}_{2}$ monol 3
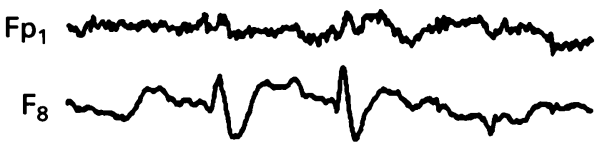

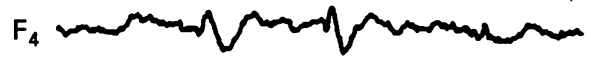
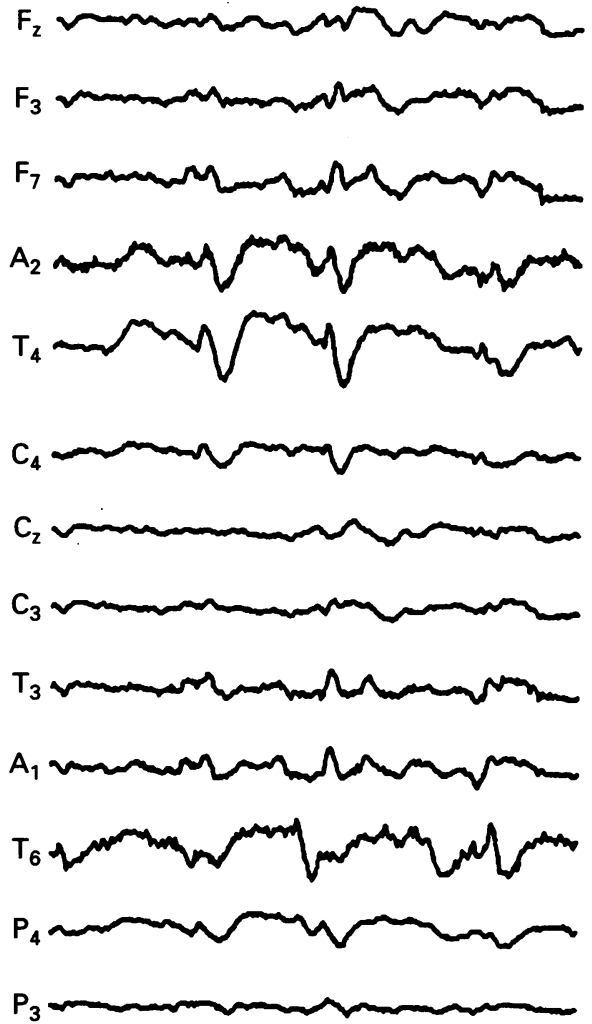

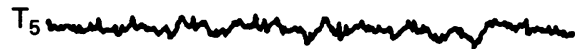
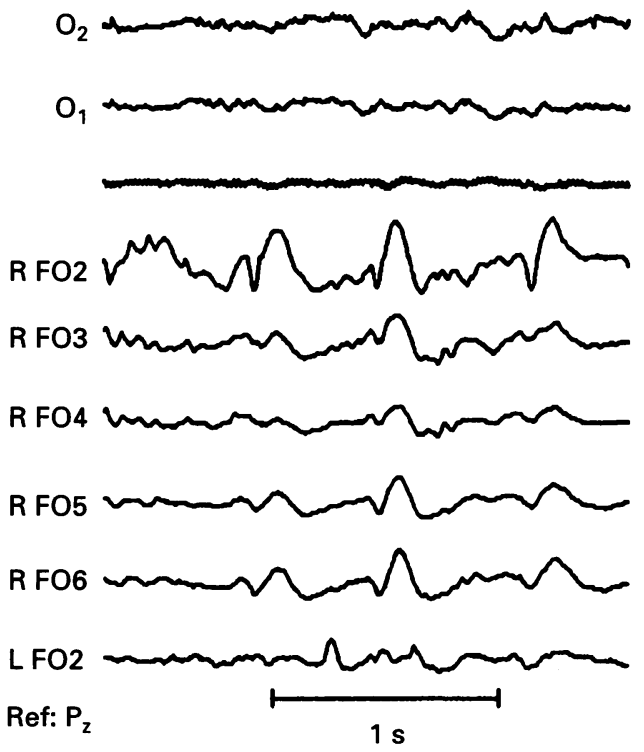

Figure 2 Examples of DO and Synch interictal activity in patient 3. Three spikes are successively recorded in the right FO contacts. The first two are associated with a clear spike and wave on the scalp and there are no time delays between FO and scalp spikes (Synch). The third spike is not associated with a clear scalp correlate (DO). Note that the first and third spikes show completely different scalp activity even though their spatial and temporal distribution in the FO electrodes are nearly identical. The calibration mark corresponds to $800 \mu \mathrm{V}$ in channels of relative gain of 1 . 
Figure 3 Two types of interictal activity in patient 6. (A) Example of DO and $S L$ interictal activity: spikes on the scalp $(A 2, T 4$, and $T 6)$ show 15-20 ms delays with respect to RFO1 and RFO2. Note there is a first spike involving RFO1 and RFO2, associated with no obvious scalp epileptiform activity, and with amplitude in the same range as the second, $S L$ spike. (B) Type LL interictal activity: spikes in deep contacts are followed by ipsilateral frontal slow waves. Note that surface recordings show a small notch nearly simultaneous with spikes in the FO electrodes. Careful latency analysis, however, showed 20 ms delays between $F O$ spikes and scalp notches. Note that FO spikes in the same range of amplitudes can be associated with no deflection, a small deflection, or a proper spike on the scalp. Calibration marks correspond to $1000 \mu \mathrm{V}$ in channels of relative gain of 1 .

Figure 4 Example of Synch interictal activity (patient 2). Synchronous events in surface and depth. All channels that record the event show the same components although with different amplitude andlor polarity. The calibration mark corresponds to $1000 \mu \mathrm{V}$ in channels of relative gain of 2 .

\section{A}

$\mathrm{Fp}_{2}$ w renary 5 $\mathrm{Fp}_{1}$ mor coneryom $F_{8}$ und remany

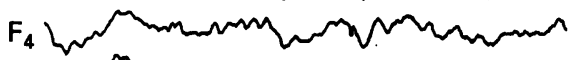

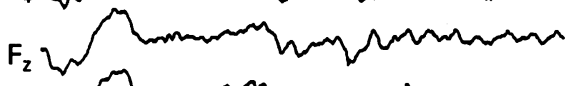
$F_{3}$ w

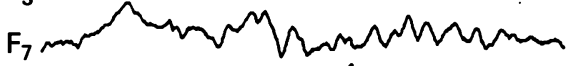

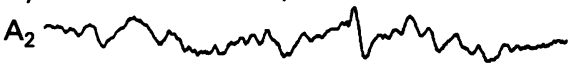

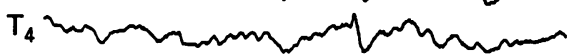

$C_{4}$

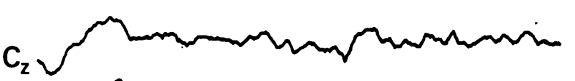
$C_{3}$ r

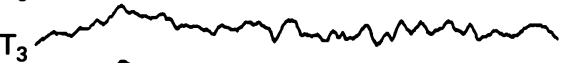

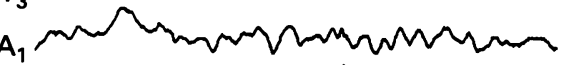
$T_{6}$ m $P_{4}$ 促

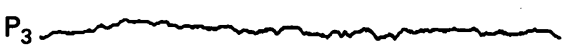

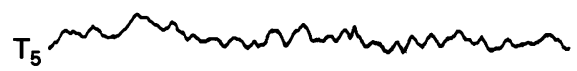

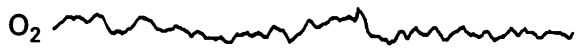

$\mathrm{O}_{1} \mathrm{Crm}$ FO1 1

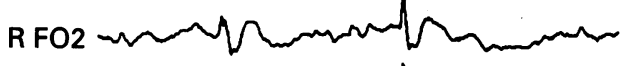

$\mathrm{ROO3}$

$\mathrm{RO} 4$

$\mathrm{RO}$

$\mathrm{RO}$ FO

LFO6 r

Ref: $P_{z}$

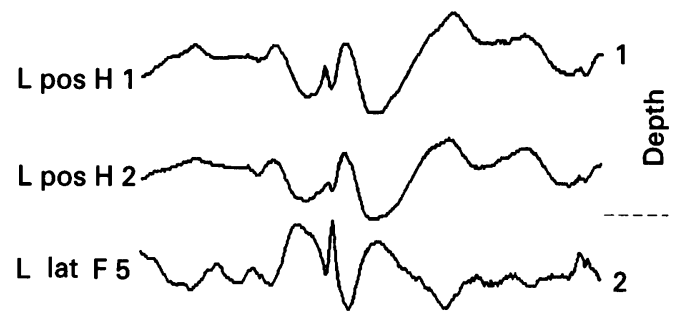

$L$ lat F7 7 ר

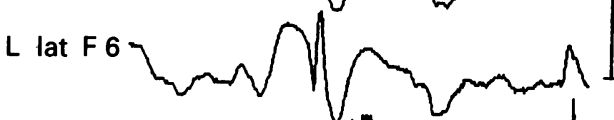

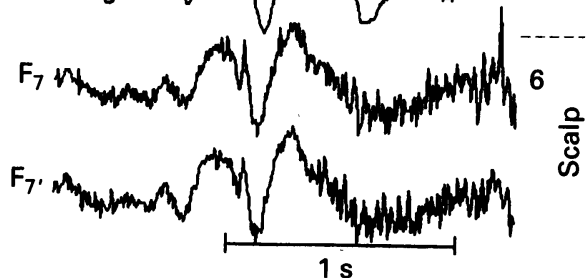

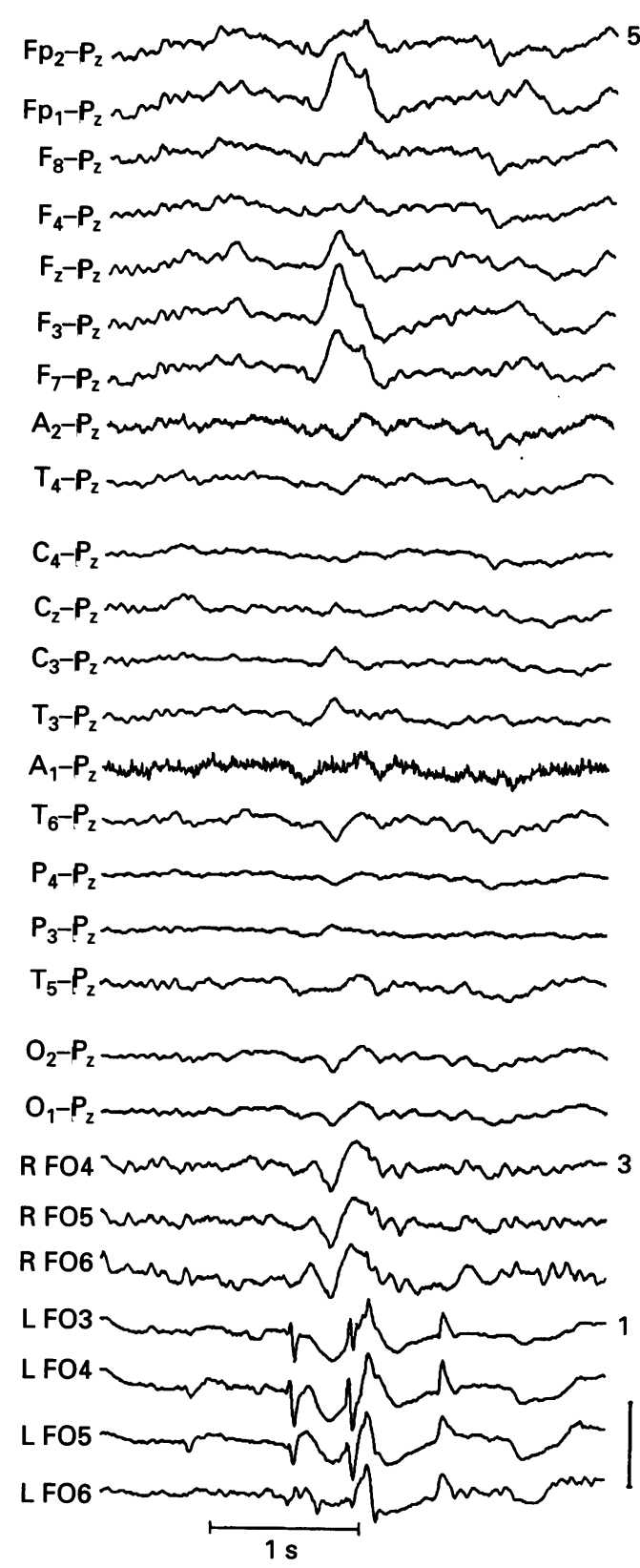

low amplitude surface recordings with different morphology from deep events, such as the onset of low amplitude surface rhythmic activity coincident with deep spikes or runs of sharp waves (see figs 1 and 8).

Type V: events recorded only by subdural or scalp electrodes (surface only, "SO", fig 7)

In this type of event, interictal epileptiform activity was recorded only by surface electrodes. Latency differences of the ranges described above could be seen between surface electrodes (fig 7). This type was recorded in two patients.

Complex mixtures of the types described were often found within the same interictal event. For instance, different ranges of latency differences were often found between deep and surface recordings, among deep recordings, or among surface traces (figs 5, 6, and 7). The table shows the different types of temporal relations present in each patient. 
Figure 5 Two examples of $L L$ interictal activity from patient 10. Spike and wave activity in left anterior and posterior hippocampus is followed, after a $105 \mathrm{~ms}$ delay, by sharp waves in left and right frontal depth electrodes and in left lateral frontal subdural electrodes. Calibration mark corresponds to $1000 \mu \mathrm{V}$.

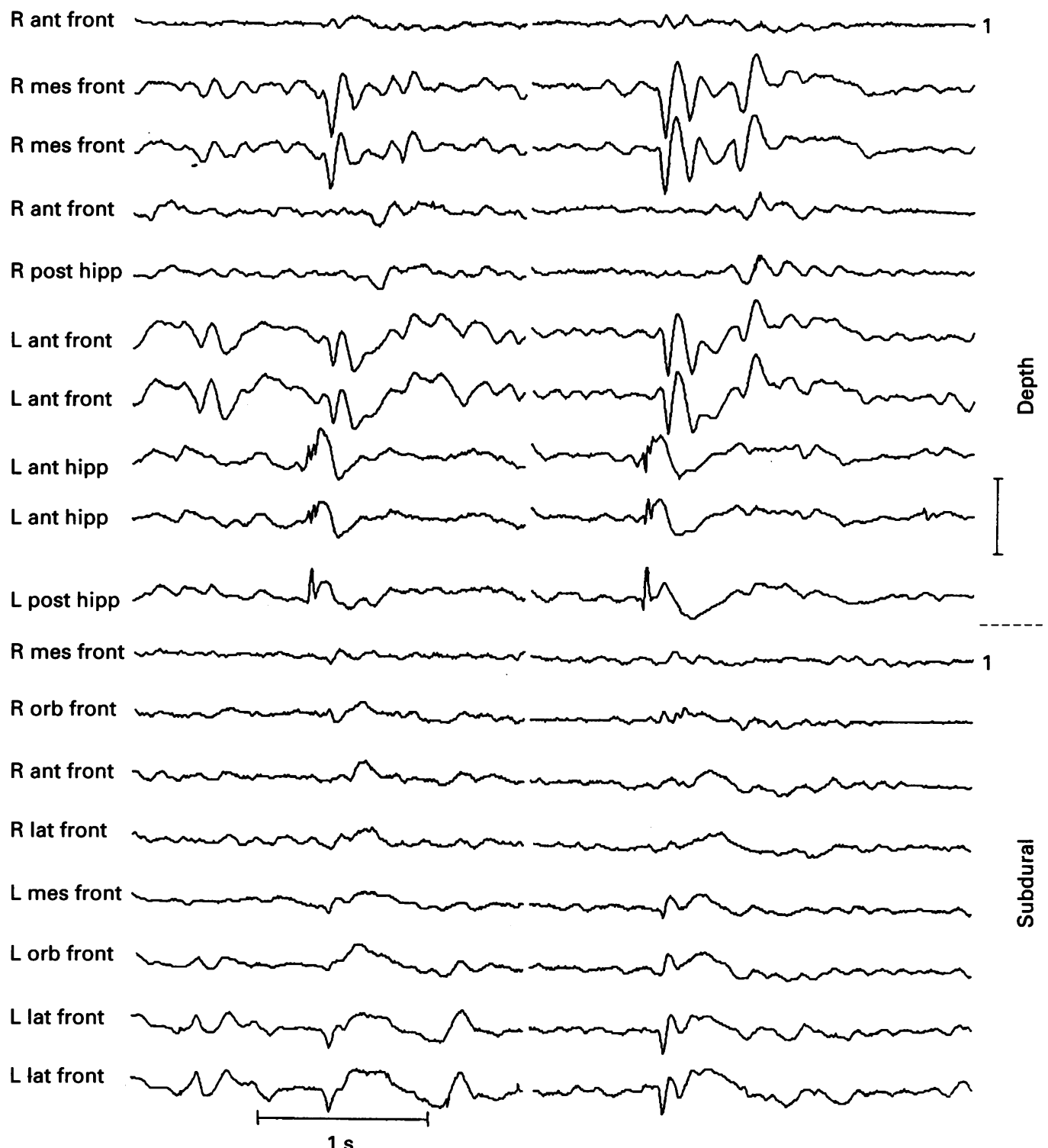

AMPLITUDE DISTRIBUTIONS IN DEPTH AND SURFACE

Interictal spikes showed average maximal amplitudes of $595 \mu \mathrm{V}$ (SD $263 \mu \mathrm{V}$ ) when recorded by intracerebral electrodes, 590 (SD 338) $\mu \mathrm{V}$ by FO electrodes, 514 (SD 304) $\mu \mathrm{V}$ by subdural electrodes, and 186 (SD 84) $\mu \mathrm{V}$ on the scalp. In any one event recorded at both surface and depth contacts, the ratio of subdural to depth amplitudes was 0.86 (SD 0.27 ) and the ratio of scalp to FO amplitudes was 0.35 (SD 0.17). One patient was studied simultaneously with depth, subdural, and scalp electrodes over temporal regions. Interictal spikes of the same polarity and frequency characteristics (apart from superimposed muscle activity) were recorded by subdural and scalp electrodes in this patient, and the ratio of scalp to subdural amplitudes was $0 \cdot 22$.

LATERALITY CORRELATIONS BETWEEN SURFACE AND DEPTH WITHIN THE SAME EVENT

Of all events recorded by surface electrodes, most $(87 \cdot 6 \%)$ were unilaterally recorded by surface electrodes and ipsilateral deep electrode(s), regardless of whether the FO or intracerebral recording protocol was used. In only $0.87 \%$ of all events was the surface activity recorded exclusively contralateral to deep activity. In $3.4 \%$ of events activity was unilateral in deep electrodes and bilateral on the surface. In $3.9 \%$ of the events, activity was recorded bilaterally in deep electrodes and unilaterally on the surface and in $4.3 \%$ of events activity was recorded bilaterally by deep and surface electrodes.

DIPOLAR DISTRIBUTION IN THE DEPTH

Patient 1 often showed phase reversals between two consecutive intracerebral channels, which suggested the existence of a single dipole generator adjacent to the intracerebral electrodes (interictal spikes recorded with opposite polarity by two contiguous intracerebral contacts and decreasing in amplitude with distance). When this was the case, subdural electrodes never recorded interictal activity associated with events that showed this type of distribution in the depth. These spikes showed largest amplitude at the left anterior hippocampus contact 5, with typical peak amplitudes of around $-500 \mu \mathrm{V}$ with respect to a remote scalp reference. They were 
Figure 6 Neocortical propagation of interictal activity (patient 11).

Polymorphic spikes recorded in the right amygdala and anterior hippocampus show synchronous onset. Negative spikes are recorded by anterior temporal subdural electrodes, simultaneous with positive spikes in posterior temporal regions, with no significant delay with respect to deep spikes. Delays, however, of over $200 \mathrm{~ms}$ are present in spikes/sharp waves

recorded in the lateral and orbital aspects of the frontal lobe and in the superior contacts of the anterior and posterior temporal electrodes, which are possibly lying over parietal regions. Note how a wave of activity propagates along the orbitofrontal contacts and the anterior temporal contacts 4 to 2, providing evidence for

intraneocortical propagation of epileptiform activity. All waves of delayed activity are preceded by small amplitude notches of variable delays. Note that spikes recorded by depth electrodes show shorter duration and higher frequency components than activity recorded by subdural electrodes. The calibration mark corresponds to $800 \mu \mathrm{V}$ in channels of relative gain of 3 .
Ramy 2

3 amy 3 3

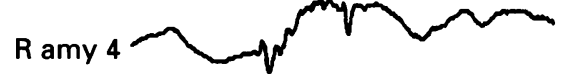
Ramy 5 Ramy 6 $\mathrm{R}$ ant H2 ${ }_{\mathrm{R} \text { ant } 3}$ $\mathrm{R}$ ant H4 $\int \frac{5}{\text { 임 }}$ $\mathrm{R}$ ant $\mathrm{H}_{5}$
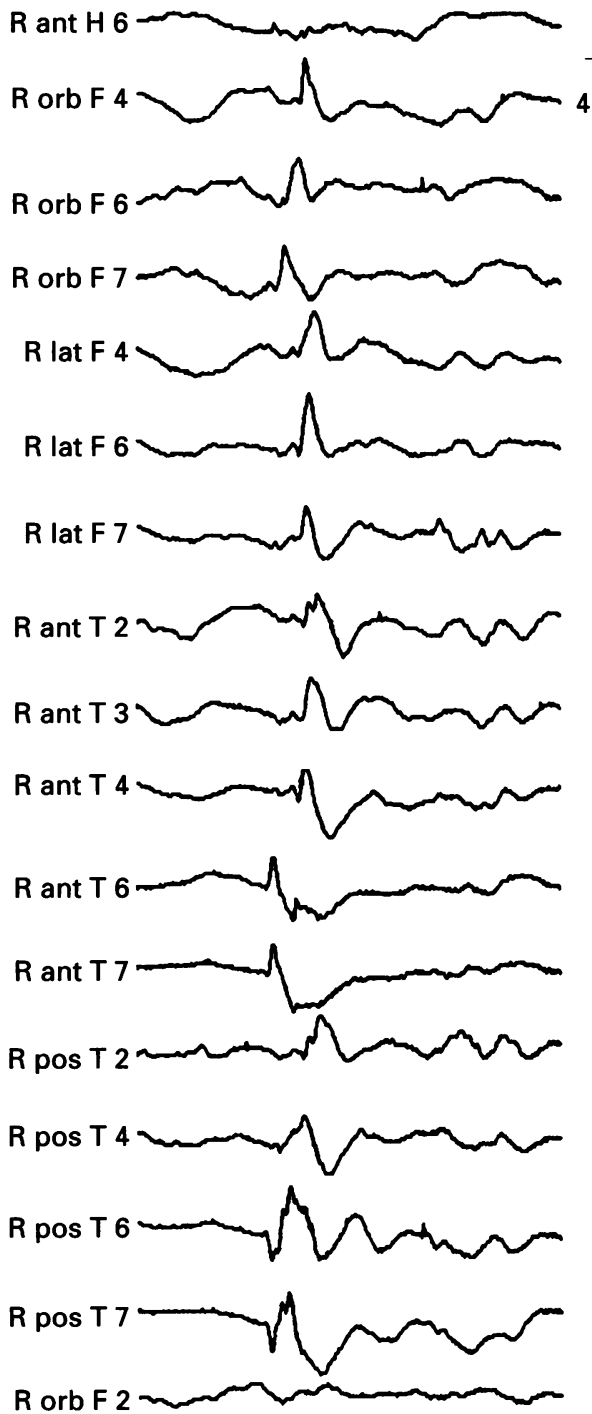

R orb $F$

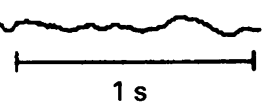

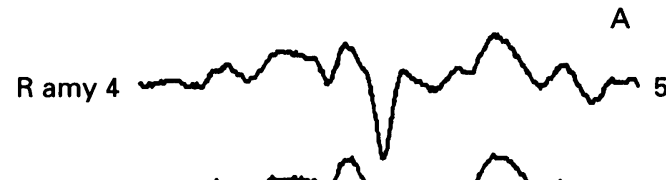

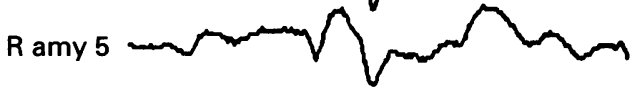

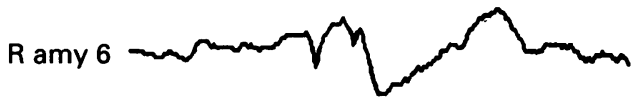

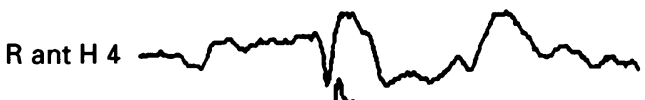

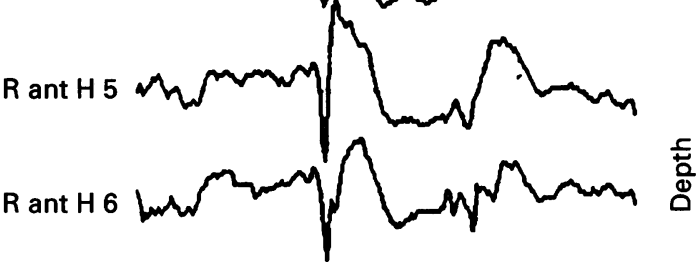

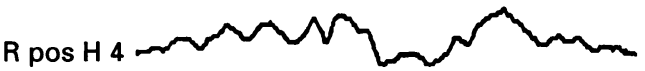

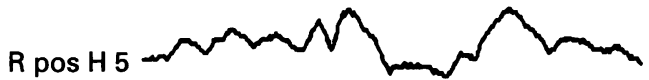

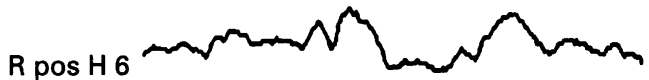

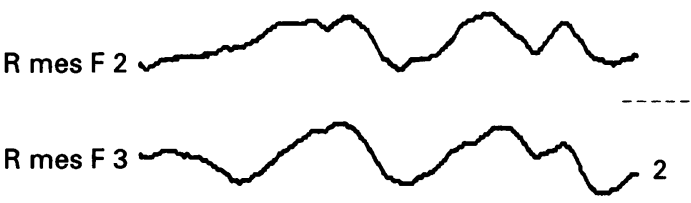

R ant T 5
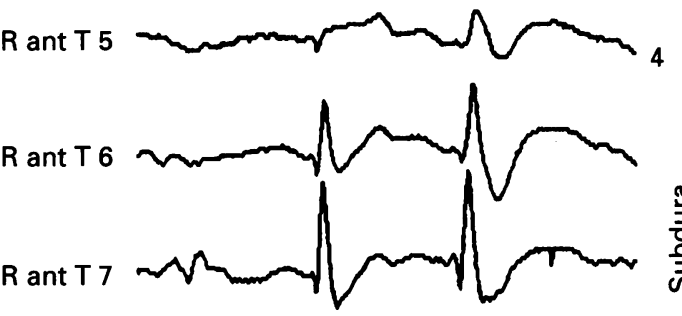

$\frac{\text { कू }}{\frac{5}{2}}$
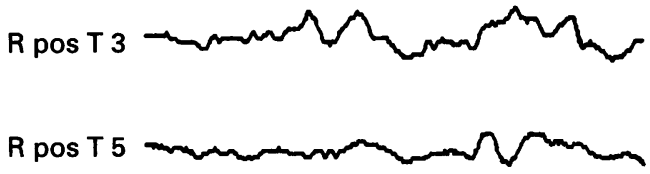

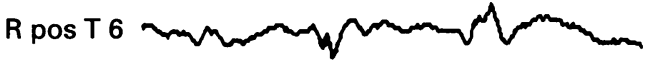

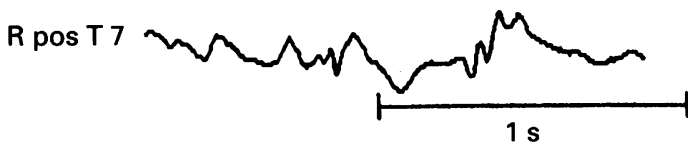

Figure 7 Example of $L L$ and $S O$ interictal discharges (patient 8). (A) Two spikes are consecutively recorded by the right anterior temporal subdural electrodes. The calibration mark corresponds to $500 \mu \mathrm{V}$ in channels of relative gain of 4. ( $B$-see opposite page) Same event expanded in time: The first spike is recorded in the right anterior hippocampus and shows no significant delays among channels at our sampling rates. The second spike, however, propagates along the right anterior temporal electrodes that are situated $2.5 \mathrm{~mm}$ apart from each other and is not clearly recorded in the depth. Note how amplitude and sharpness decrease as the spike propagates along the subdural electrodes, perhaps reflecting gradual synchronicity loss. 
Fig $7 B$
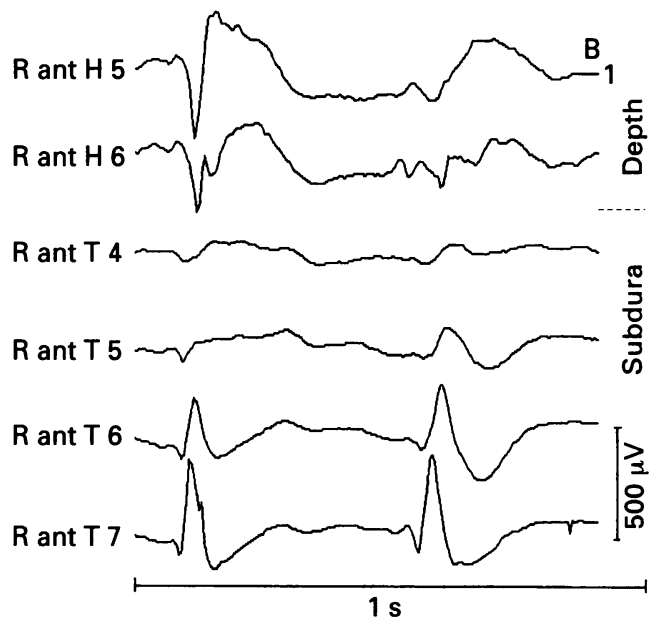

recorded with opposite polarity and half the amplitude (about $250 \mu \mathrm{V}$ ) at contact 6 , situated $2.5 \mathrm{~mm}$ inferior to contact 5 .

\section{FREQUENCY SPECTRA OF SPIKES AND}

PRECEDING RHYTHMIC ACTIVITY

In interictal activity type DO, SL, and LL, spikes recorded by deep electrodes often showed shorter duration and sharper waveforms than surface events (figs $1,4,5,6,7$, and 8). Surface events usually consisted of negative sharp/slow waves or negative spikes followed by a slow wave of opposite polarity. Interictal events recorded in the depth were usually sharper spikes, often polyphasic, with several consecutive sharp deflections (figs 5 , 6,7 , and 8 ).

\section{CORRELATION BETWEEN INTERICTAL AND}

ICTAL FOCI

The relations in topographies of ictal onset foci and interictal foci (sites where interictal spikes were recorded) were studied. In all patients in whom a single ictal onset zone was found in the depth (five patients with intracerebral electrodes and two with FO electrodes) the electrodes that recorded the onset of ictal activity also recorded frequent interictal spikes. In all seven patients, however, there were also frequent independent interictal spikes in at least one deep electrode contralateral to the site of onset of seizures. Recorded surface interictal events could be associated with any of the deep interictal foci, regardless of their capacity to trigger seizures. In three patients, surface interictal spikes that were recorded independently on either side had associated activity recorded at the ipsilateral deep electrodes. In three patients only the deep interictal spikes that were seen at the site of onset of seizures were associated with interictal activity on the surface. In one patient, however, interictal events recorded by surface

Table For each patient is shown the topography of interictal events analysed by computer, the presence of different types of delays, the number of interictal events analysed, the number of seizures recorded during telemetry, and the ictal onset as suggested by ictal telemetry recordings

\begin{tabular}{|c|c|c|c|c|c|c|c|c|c|}
\hline $\begin{array}{l}\text { Patient } \\
\text { number }\end{array}$ & $D O$ & Synch & $S L$ & $L L$ & SO & $\begin{array}{l}\text { Topography } \\
\text { interictal }\end{array}$ & $\begin{array}{l}\text { Number } \\
\text { interictal }\end{array}$ & $\begin{array}{l}\text { Number } \\
\text { seizures }\end{array}$ & $\begin{array}{l}\text { Seizure } \\
\text { onset }\end{array}$ \\
\hline 1 & + & $\begin{array}{l}\mathrm{d} \\
\mathrm{s}\end{array}$ & ds & - & - & $\begin{array}{l}\text { R AMY, R, PT, R AT } \\
\text { L AH }{ }^{\star}, L \text { PH }^{\star}\end{array}$ & 4 & 4 & L PH \\
\hline 2 & - & $\begin{array}{l}\text { d } \\
\text { s } \\
\text { ds }\end{array}$ & $\mathbf{s}$ & $\mathbf{s}$ & - & $\begin{array}{l}\text { L AMY, L AH, L PH } \\
\text { L MF, L LF, L OF, } \\
\text { L AT, R AMY* }\end{array}$ & 4 & 6 & $\begin{array}{l}\text { L PH } \\
\text { L AH }\end{array}$ \\
\hline 3 & + & $\begin{array}{l}\text { d } \\
\text { s } \\
\text { ds }\end{array}$ & $\mathrm{d}$ & - & - & $\begin{array}{l}\text { R FO, F8, F4, T4, } \\
\text { T6, LFO F }\end{array}$ & 5 & 6 & nk \\
\hline 4 & + & $\begin{array}{l}\text { d } \\
\text { s }\end{array}$ & ds & - & - & $\begin{array}{l}\text { L FO, Fp2, F8, T6 } \\
\text { R FO` }^{\star}\end{array}$ & 2 & 7 & $\begin{array}{l}\text { L FO } \\
\text { R FO }\end{array}$ \\
\hline 5 & + & - & - & - & - & R FO, L FO* & 3 & 3 & R FO \\
\hline 6 & + & $\begin{array}{l}\text { d } \\
\text { s } \\
\text { ds }\end{array}$ & $\begin{array}{l}\text { d } \\
\text { s } \\
\text { ds }\end{array}$ & ds & - & 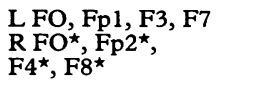 & 4 & 1 & nk \\
\hline 7 & + & $\begin{array}{l}\text { s } \\
\text { ds }\end{array}$ & d & $\begin{array}{l}\text { ds } \\
\text { ds }\end{array}$ & - & R FO, F8, Fp2, T4, & 3 & 4 & $\mathrm{R} F O$ ? \\
\hline 8 & + & $\begin{array}{l}\text { d } \\
\text { s } \\
\text { ds }\end{array}$ & $\begin{array}{l}\text { s } \\
\text { ds }\end{array}$ & - & + & $\begin{array}{l}\text { R AH, R AT } \\
\text { LAMY }{ }^{\star}, \mathrm{LH}^{\star}\end{array}$ & 5 & 3 & R AH \\
\hline 9 & + & $\begin{array}{l}\text { d } \\
\text { s } \\
\text { ds }\end{array}$ & $\begin{array}{l}\text { s } \\
\text { ds }\end{array}$ & - & + & $\begin{array}{l}\text { R AMY AM, L AH, } \\
\text { L PH, L AT, L PT, } \\
\text { L OF }\end{array}$ & 4 & 4 & $\mathrm{LPH}$ \\
\hline 10 & + & $\mathrm{d}$ & - & $\mathrm{d}$ & - & $\begin{array}{l}\text { R MF, L, MF, L AH, } \\
\text { L PH, L OF, L LF }\end{array}$ & 4 & 4 & $\mathrm{mf}$ \\
\hline 11 & + & $\begin{array}{l}\text { d } \\
\text { s } \\
\text { ds }\end{array}$ & - & $\begin{array}{l}\text { d } \\
\text { ds }\end{array}$ & - & $\begin{array}{l}\text { R AMY, R AH, R OF } \\
\text { R LF, R AT, R PT } \\
\text { L AMY* }\end{array}$ & 4 & 6 & $\begin{array}{l}\text { R AMY } \\
\text { R AH }\end{array}$ \\
\hline 12 & + & $\begin{array}{l}\mathrm{d} \\
\mathrm{s}\end{array}$ & $\begin{array}{l}\text { d } \\
\text { ds }\end{array}$ & ds & - & 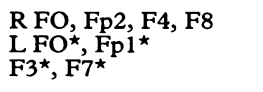 & 3 & 5 & L FO \\
\hline
\end{tabular}

DO = deep only; Synch = synchronous; $S L=$ short latency; $L L=$ long latency; SO = surface only; $+=$ present; $d=$ present among deep recordings; $s=$ present among surface recordings; ds = present between deep and surface recordings; $-=$ not present; $\mathrm{R}=$ right; $\mathrm{L}=$ left; FO = Foramen ovale electrodes; nk = not known; mf = multifocal; INTRACEREBRAL ELECTRODES: $\mathrm{R}=$ right; $\mathrm{L}=$ left; $\mathrm{FO}=$ Foramen ovale electrodes; $\mathrm{nk}=$ not known; $\mathrm{mf}=$ multifocal; INTRACEREBRAL ELECTRODES:
$\mathrm{AMY}=$ amygdala; AH = anterior hippocampus; $\mathrm{PH}=$ posterior hippocampus; $\mathrm{MF}=$ mesial frontal; SUBDURAL ELECTRODES: $\mathrm{PT}=$ posterior temporal; $\mathrm{AT}=$ anterior temporal; $\mathrm{LF}=$ lateral frontal; $\mathrm{OF}=$ orbitofrontal; independent intericELECTRODES: $\mathrm{PT}=$ posterior temporal; $\mathrm{AT}=$ anterior
tal events are marked with different numbers of asterisks. 
Figure 8 Interictal spike/sharp wave preceded by rhythmic build up (patient 12). The scalp spike is preceded by a 300 ms rhythmic activity at 12 Hz. This scalp rhythmic activity coincides with a 16 Hz rhythm at the LFO2 and LFO3 that halts about $200 \mathrm{~ms}$ before the main scalp spike. Note that the scalp spike coincides with a polymorphic sharp wave at LFO2 and LFO3, and smoother sharp waves at LFO5 and LFO6. The calibration mark corresponds to $600 \mu \mathrm{V}$ in foramen ovale recordings. electrodes were associated with spikes recorded only by deep electrodes contralateral to the ictal onset zone, even though frequent interictal spikes were recorded independently by deep electrodes at the ictal onset focus.
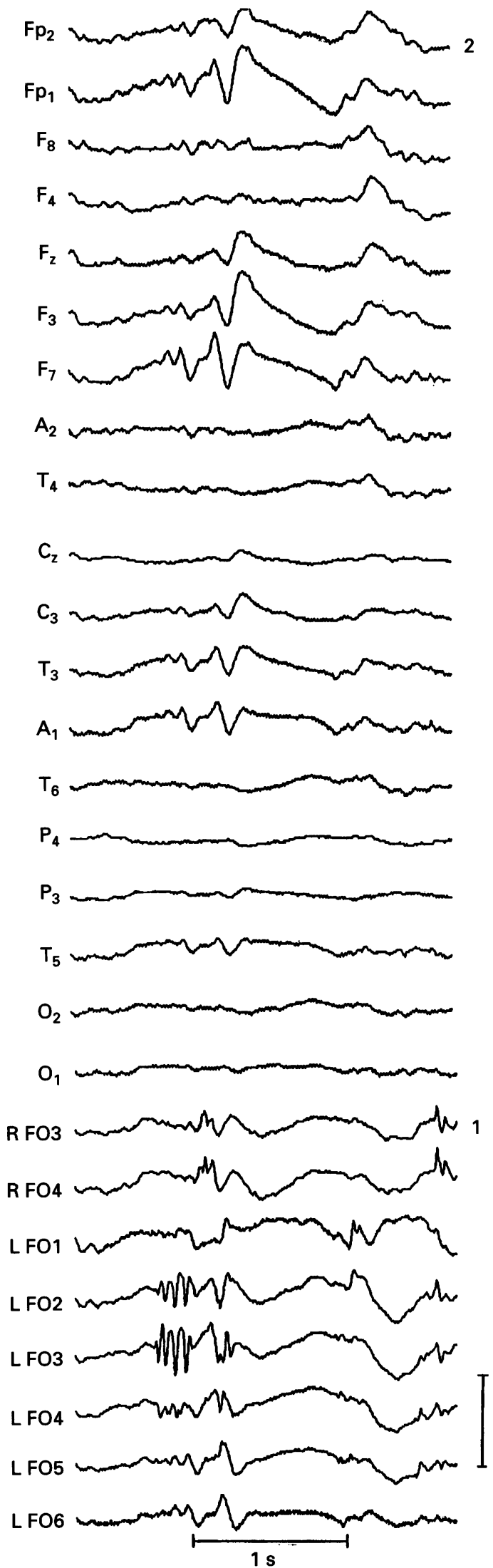

Ref: $\mathbf{P}_{\mathbf{z}}$
ANALYSIS OF ICTAL ACTIVITY

Electrical ictal events in complex partial seizures often remained confined to a limited number of deep contacts for a few seconds before spikes are recorded on the surface. Onset of seizures is often associated with the appearance of rhythmic electrical activity in deep recordings which can be largely characterised by a single frequency. Because electromagnetic fields propagate with the speed of light, an increment in the power of any oscillator should be associated with an instantaneous increase in the power of the oscillation frequency at all recording sites where the signal from the oscillator is above noise levels. Visual inspection and spectral analysis showed that when a single ictal onset zone was found, large amplitude frequency components appearing at ictal onset in the deep contacts were not present above noise levels in surface contacts for several seconds. Figure 9 gives an example. Although Fourier analysis provides a strong additional tool in the characterisation of rhythmic phenomena, it has not been commonly applied to the estimation of volume conduction at seizure onset. A more detailed analysis of ictal activity in 15 patients will be reported elsewhere.

\section{Discussion}

The results presented in this study suggest that different neural populations may be sequentially or simultaneously active at relatively widely separated locations during interictal paroxysmal events. In general, there is no consistent relation between depth and surface recordings, and in most cases no single source can account for the temporal or spatial distribution of depth and surface potentials. Phase reversals in deep recordings compatible with dipolar generators are rarely recorded. The spatial distribution of voltage in deep recordings is typically non-dipolar, showing spikes in the same range of amplitudes in two or three contiguous electrodes and suddenly disappearing in the next electrode, situated only a few millimetres apart, perhaps reappearing in other electrodes further away. Moreover, interictal epileptiform events are often not recorded simultaneously by deep and surface electrodes, although showing specific spatiotemporal patterns within patients, suggesting that epileptiform activity often propagates quickly along neural pathways. Furthermore, spikes recorded by intracerebral and subdural electrodes show the same range of maximal amplitudes, and voltage gradients recorded from deep structures (less than 1000 $\mu \mathrm{V} / 2.5 \mathrm{~mm}$, with equivalent dipoles in the order of $2 \mathrm{nA} \cdot \mathrm{m}$ ) cannot account for signal amplitudes recorded by subdural or scalp electrodes, or for the strength of hypothetical deep dipoles calculated from scalp MEG or EEG recordings (in the order of $300-600$ $\mathrm{nA} \cdot \mathrm{m})$.

\section{LATENCY DIFFERENCES}

The largest proportion of interictal activity is recorded only by deep electrodes. Often deep 
A

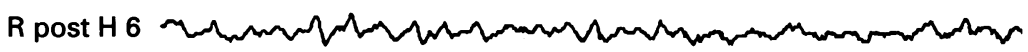

$\mathrm{R}$ ant $\mathrm{H} 4$

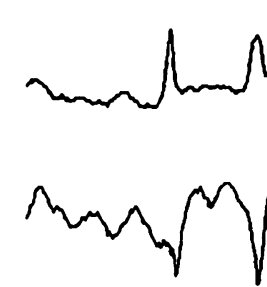

$\mathrm{R}$ ant $\mathrm{H} 5$

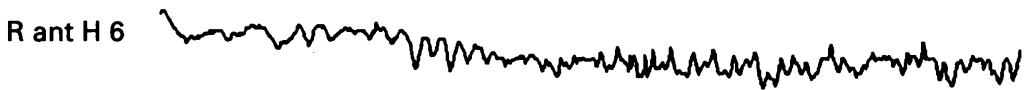

R ant $\mathrm{H} 6$
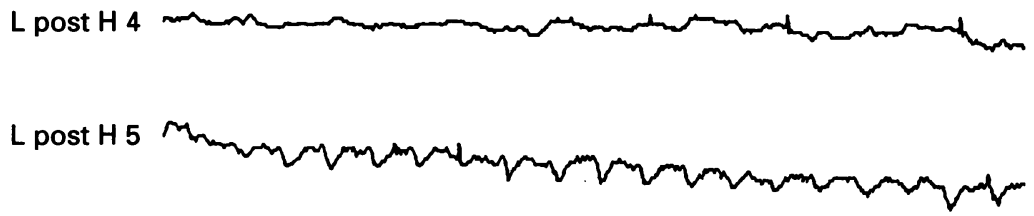

L post $\mathrm{H} 6$

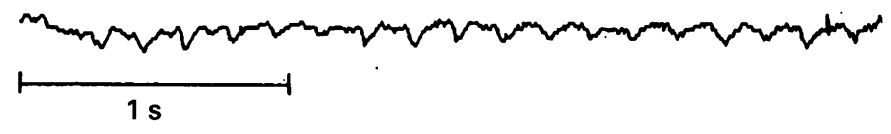

B

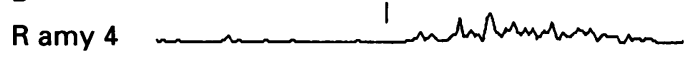

R amy 5

Ramy 6 Ansprythen

$\mathrm{R}$ ant $\mathrm{H} 4$

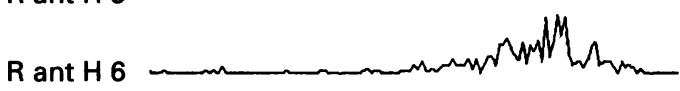

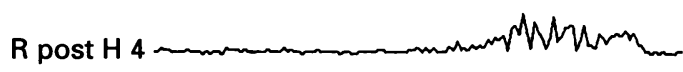

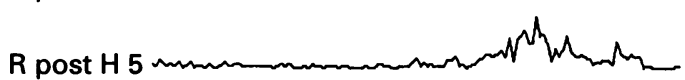

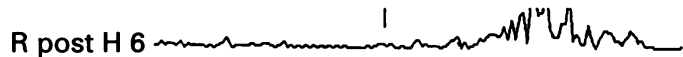

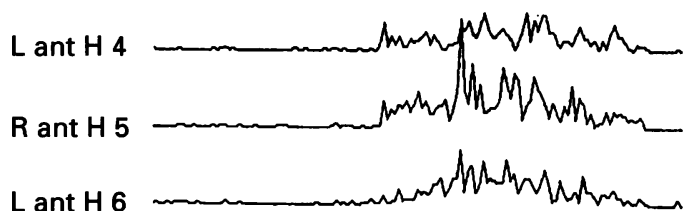

Figure 9 Analysis of ictal activity in patient 1. (a) Onset of rhythmic ictal activity on the left anterior hippocampus (contacts 4 and 5). The peak to peak amplitude on contact 5 is $360 \mu \mathrm{V}$ during the first nine cycles. Power analysis revealed the main frequency of the rhythmic transformation at $14 \cdot 8$ $( \pm 0 \cdot 39) \mathrm{Hz}$. (b) Time evolution of the amplitude at $14.8 \mathrm{~Hz}$ of the EEG Fourier transform calculated for consecutive epochs of $1 \cdot 28$ sec recorded during 4 minutes, which included preictal, ictal and postictal activity. The onset of ictal activity coincides with the vertical mark. Note that the increase in $14 \cdot 8$ $\mathrm{Hz}$ activity which characterises seizure onset remains localised to left anterior hippocampus for about 15-20 seconds. As volume conduction propagates instantaneously within the brain, this finding indicates that significant amounts of ictal activity reach the surface only after neural propagation.

events with similar amplitudes, or even waveforms, occur both with and without significant accompanying surface signals (figs 2 and 4). Therefore, events recorded only by deep electrodes must represent neuronal activity that does not propagate significantly through neural pathways to the gyri.

More direct evidence of neuronal propagation of interictal activity from deep structures to the neocortices in humans is provided by time delays between deep and surface recordings. Delays of as much as $220 \mathrm{~ms}$ between deep interictal spikes and surface activity have been measured. The shortest delays (SLs) are associated with surface spikes, whereas longer latency differences (LLs) are associated with surface sharp or slow waves, often preceded by low amplitude rhythmic activity or a small deflection. Because the main waveform is usually the same in deep and surface SL activity, delays in the range of $10-50 \mathrm{~ms}$ could in principle be explained by: (1) the filtering behaviour of the tissues due to their passive electrical properties (resistance, capacitance) in the presence of a single source closer to deep electrodes; and (2) different channels recording different electromagnetic sources that correspond to neural populations that share a generic form of activation in response to a common epileptogenic event. The filtering behaviour of the tissues seems to be negligible at the frequencies of interest. It has been shown that the cerebral white matter behaves predominantly in a resistive manner up to $20 \mathrm{kHz}^{20}$ and the dielectric permittivity and electrical conductivity of fresh and fixed bone are independent of frequency up to $100 \mathrm{kHz} .^{21}$ Alternatively, different cortical regions could be activated nearly simultaneously by fast conductive fibres from a deep source, presumably close or within mesial temporal structures but perhaps from other subcortical or limbic structures. In any one SL interictal spike, all channels that record the event often show a similar pattern consisting of a negative or positive spike followed by a slow wave of opposite polarity, each component showing different duration and sharpness, presumably reflecting differences in cell synchronisation.

Longer latency interictal activity provides clear evidence for neural propagation of activity from deep sources to the neocortex. Sharp or slow waves are recorded 50 to $220 \mathrm{~ms}$ after a deep spike. Neither the waveform nor the large delay, nor the fact that delayed activity often has larger amplitude than early spikes, can be explained by tissue filtering. Deep interictal spikes often occur, however, with low amplitude deflections or rhythmic activity recorded on the surface preceding the main sharp wave (figs $1,4,6$, and 8). These initial low amplitude deflections show SL and LL delays, which suggest that they could represent a volley of arriving activity that triggers local phenomena responsible for the generation of late sharp waves (Figs 1 and 7).

In seven patients, a proportion of interictal spikes showed no delays between deep and surface electrodes (Synch). The lack of delay could in principle be explained by a single source. Amplitude inconsistencies between deep and surface recordings exist, however 
Figure $9(B)$ continued.

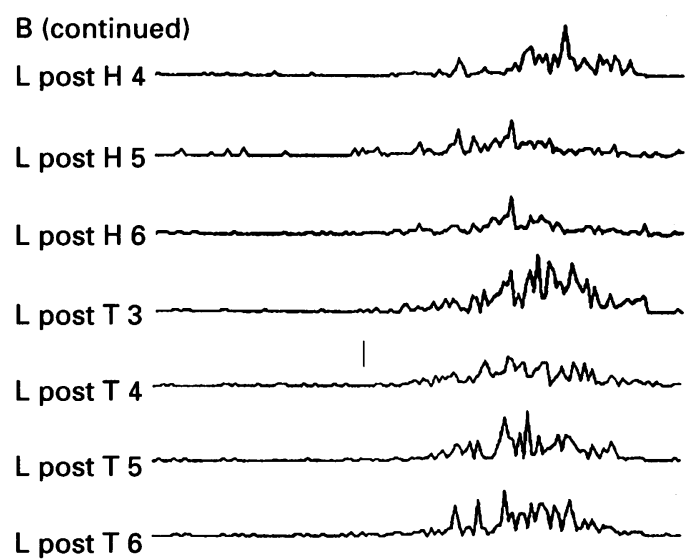

$R$ ant $T 5$ post $T 7$
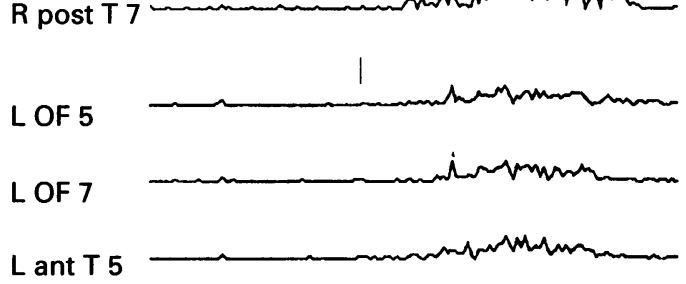

L ant T 7

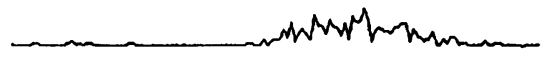

L MT 5

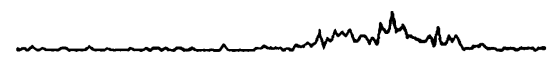

L MT 7

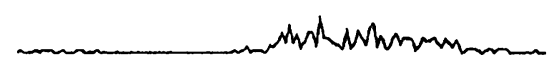

L Pa 5

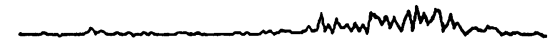

L Pa 7

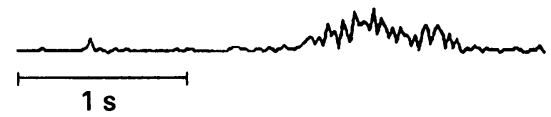

(see later). Alternatively, as with SL, Synch activity could also represent a generic mechanism of cortical activation by fast conducting fibres driven by deep sources or by a structure from which activity has not been recorded.

SO interictal activity (recorded only on the surface) may represent events only involving neocortex, or those in which deep activity, if present, has not been detected in depth recordings.

\section{AMPLITUDE DISTRIBUTION}

Even when latency differences are compatible with a single deep source, amplitude inconsistencies exist. There is no clear proportionality between amplitude distributions in depth and surface within the same patient. Nearly identical interictal events in the depth can accompany completely different events on the surface (figs 2 and 4 ) and vice versa (fig. 7). The spatial distribution of voltage in deep recordings is usually not clearly dipolar, often showing spikes in the same range of amplitudes at two or three contiguous electrodes, abruptly disappearing at the next electrode, situated only a few millimetres apart, perhaps reappearing at other electrodes further away. The topography of interictal paroxysms is quite localised in the depth, often recordable over no more than one or two centimetres, yet showing a relatively wide distribution and high amplitude on the surface (fig 2). Also, depth and subdural interictal spikes show the same range of amplitudes. These features of spatial distribution suggest that neural populations located as far as several centimetres apart should be simultaneously active during interictal activity, a situation that could severely hamper space discrimination in dipole source localisation.

Also, phase reversals in deep recordings compatible with the existence of deep dipoles are rare. In our series some interictal activity in only one patient showed dipolar distribution in the depth, with voltages of about $750 \mu \mathrm{V}$ between two hippocampal electrodes situated $2.5 \mathrm{~mm}$ apart. Assuming grey matter resistivity of $2.84 \mathrm{ohm} \cdot \mathrm{m}^{19}$ and assuming that the measured voltages were generated by a dipole located in the hippocampus between both electrodes-for example, $1 \mathrm{~mm}$ from contact 5 , the current dipole in the depth would have to be of the order of $2 \mathrm{nA} \cdot \mathrm{m}$. The largest voltages generated at the subdural space or on the scalp (situated about 4 and $5 \mathrm{~cm}$ from mesial temporal structures) by a $2 \mathrm{nA} \cdot \mathrm{m}$ current dipole would be of the order of 0.7 and $0.45 \mu \mathrm{V}$ respectively (assuming white matter resistivity $\left.=6 \cdot 82 \mathrm{ohm} \cdot \mathrm{m}^{19}\right)$. Similarly, assuming the magnetic permeability of human tissues of the same order as the magnetic permeability of free space, the largest magnetic fields generated over the scalp by a current dipole of $2 \mathrm{nA} \cdot \mathrm{m}$ situated in mesial temporal structures would be $88 \mathrm{fT}$. Therefore, EEG amplitudes measured on the surface are three orders of magnitude larger than predicted by a single dipole situated in mesial temporal structures, with strength compatible with amplitudes generated in the depth. Similarly, measured MEG scalp spikes (about $1 \mathrm{pT}$ ) are one order of magnitude bigger than predicted.

INCONSISTENCIES BETWEEN THE AMPLITUDE OF DEEP EPILEPTIFORM ACTIVITY, THE STRENGTH OF DEEP DIPOLES CALCULATED FROM SCALP MEASUREMENTS AND THE AMPLITUDE OF SCALP SIGNALS PRODUCED BY DEEP IMPLANTED DIPOLES

Several studies with deep implanted electrodes and simultaneous subcortical, cortical, and scalp recordings have shown that potential differences due to deep dipoles are attenuated by over 1:5000 on the scalp and are undetectable in the EEG unless dipole voltages are much higher than those ever found in brain tissue. It was concluded that volume conduction inside the brain is very small. ${ }^{22}{ }^{23}$ Recent work has shown that deep implanted dipoles require highly supraphysiological current intensities to generate relatively small signals on the scalp. For instance, current dipoles of the order of $320 \mathrm{nA} \cdot \mathrm{m}$ (the 
maximum allowed by safety regulations in the Massachusetts Institute of Technology) were required to produce $15 \mu \mathrm{V}$ deflections on the scalp. ${ }^{24}$ This means that for amplitudes of epileptiform activity reported in the present study (intracranial voltage gradients in the range of $750 \mu \mathrm{V} / 2.5 \mathrm{~mm}$, with equivalent currents of about $2 \mathrm{nA} \cdot \mathrm{m}$ and scalp amplitudes of $186 \mu \mathrm{V}$ ), the ratio of deep-to-superficial electrical activity recorded on the scalp during an interictal event would be of the order of $1: 2000$. Furthermore, the strength of hippocampal dipoles calculated from scalp MEG or EEG measurements, being of the order of 300-600 $\mathrm{nA} \cdot \mathrm{m}$, is well above currents required to generate the amplitudes of deep signals measured in this study. Dipoles of this strength would generate voltage gradients of about $80 \mathrm{mV}$ across sites situated $2.5 \mathrm{~mm}$ apart across the dipole, which are about two orders of magnitude larger than the amplitude of spikes ever recorded in the brain. These estimations suggest that signals spreading by volume conduction from deep sources are hardly recordable on the scalp. This implies that scalp EEG signals arise from generators closer to the scalp than the estimated deep dipoles. Few authors who use modelling to locate equivalent dipoles give estimates of their values. The amplitude of scalp measurements used for modelling, however, imply dipole magnitudes far in excess of the signal strength observed in depth signals-for example, a $100 \mu \mathrm{V}$ spike widely distributed over the anterior temporal region would require a mesial temporal dipole moment of about $100-600 \mathrm{nA} \cdot \mathrm{m}$.

\section{ANATOMICOPHYSIOLOGICAL BASIS OF NEURAL} PROPAGATION

The existence of mutual interconnections between amygdala and hippocampus and widespread projections from both structures to the neocortices have been well established (for reviews see Lopes da Silva et $a l^{25}$ and Pandya and Yeterian ${ }^{26}$ ). They provide an anatomical substrate to the interictal propagation of activity from deep sources to neocortex or in the opposite direction. In particular, Amaral and Price have described extensive projections from the amygdala to the pole of the temporal lobe, the superior temporal gyrus and the medial and orbital surfaces of the frontal lobe. ${ }^{27}$ This is consistent with the finding that mesial temporal spikes are often associated with lateral frontal, orbitofrontal and anterior temporal spikes or sharp waves on subdural electrodes (figs 5, 6, and 7) and with anterior temporal spikes on the scalp (figs 1, 2, 4, and 8); see also Hughes. ${ }^{28}$ Unfortunately little is known about the diameter of fibres projecting from mesial temporal structures to the neocortex and whether their conduction velocity could account for the neocortical activation by mesial temporal structures with the latency differences described (more than $3 \mathrm{~m} / \mathrm{s}$ for LL delays and 1.2 to $3 \mathrm{~m} / \mathrm{s}$ for SL). Very fast conducting rates have been found, however, in the CNS. For instance, the first cortical com- ponents of somatosensory evoked responses appear with $20 \mathrm{~ms}$ delay after medial nerve stimulation. ${ }^{29}$ Assuming a peripheral conduction velocity of about $60 \mathrm{~m} / \mathrm{s}$ for the fastest afferent fibres ( $12 \mu \mathrm{m}$ diameter A fibres), ${ }^{30}$ peripheral conduction probably takes at least $10 \mathrm{~ms}$, leaving $10 \mathrm{~ms}$ for central conduction to occur from the cervical spinal cord to somatosensory cortex. Similarly, large areas of the neocortex could be synchronised in a few milliseconds by mesial temporal structures, or archicortex and neocortex could be activated simultaneously by a limbic or a subcortical pacemaker, perhaps thalamic. In any case, at the usual sampling rates, short or absent delays are compatible with activation of temporal and frontal neocortex through fast conducting fibres from deep sources. Even for the slowest axonal conduction, however, delays over $70 \mathrm{~ms}$ require a multisynaptic pathway, presumably with intracortical or subcortical relays. We have noted that neocortical events which show long delays are often preceded by a low amplitude deflection or rhythmic activity that starts with a deep spike (figs $1,4,6$, and 8 ). This low amplitude activity could represent an initial neocortical afferent volley that is responsible for the generation of local changes that will in turn give way to a propagated sharp/slow wave under appropriate conditions.

\section{NEURAL SYNCHRONICITY}

An interesting finding is that deep recordings often show sharper and shorter spikes than surface recordings. Synchronisation could be lost as the wave travels, probably due to statistical differences in synaptic delays or fibre conduction velocities. Moreover, deep spikes can be associated with surface spikes, sharp waves, or slow activity, down to the delta range. Usually the slowest activities showed the longest delays and more widespread spatial distribution. These findings are consistent with the suggestion that sharper epileptiform activity and fast spikes are more common when recording from close to the neural generator of epileptiform activity. ${ }^{31}$

\section{NEURAL $v$ ELECTROMAGNETIC PROPAGATION}

Our results regarding latency and spatial distributions, suggest that interictal activity is a complex phenomenon in which relatively large areas of neocortex and archicortex can be simultaneously or consecutively activated through three mechanisms: (1) by fast association fibres directly, (2) by fast association fibres that trigger local phenomena which might in turn give way to sharp/slow waves or spikes, and (3) propagation along the cortex. Also, there must be a generic mechanism by which widespread areas of the cortex generate certain patterns in response to epileptiform events. Specific factors such as neuron network anatomy, dynamics, or previous state could condition the cortex to generate spikeand-wave, sharp, or slow waves as a response. The simultaneous activation of large areas of the neocortex in a generic form can simulate electrical activity distributed over a wide solid 
angle, similar to that generated by a deep single electrical source. In this context, the distinction between electromagnetic and neuronal propagation of the signal can be difficult because the existence of multiple solutions to the inverse problem complicates modelling in the presence of multiple sources. For instance it can be shown that the deeper the source the wider the surface area where it is detected..$^{32}$ Therefore, it could be difficult to distinguish scalp electromagnetic fields generated by an extended neocortical source from those generated by a single deep source on the basis of the spatial distribution of external electromagnetic fields. The magnitude of the dipole moment should also be considered, and must be compatible with physiological neuronal currents (with voltage gradients smaller than $1000 \mu \mathrm{V} / 2.5 \mathrm{~mm})$. Hence, when deep and surface sources are active simultaneously, as seems to be the case in Synch and SL types, the active sources nearest the recording sites could mask or cancel activity from deep sources, which additionally shows a very small signal to noise ratio. Averaging would probably not improve the signal to noise ratio of deep sources significantly, as simultaneous signals from superficial sources, several hundred times larger, are also time locked to the average. Under these circumstances, modelling of neuronal activity as a single dipole or as a small number of dipoles seems to be inadequate. In a situation where the contribution of volume currents from deep sources to scalp EEG is minimal and most of the surface EEG or MEG is due to the activated neocortex underlying the recording site, MEG would not provide major advantages over EEG recordings for deep source characterisation, apart from being insensitive to radial currents and the lack of patient reference. Nevertheless, a number of reports have localised scalp epileptiform MEG activity to single deep structures. As a deep temporal epileptogenic zone commonly produces, mainly propagated, interictal EEG and MEG discharges over the ipsilateral temporal neocortex which can be represented by an equivalent dipole within the temporal lobe, evidence of the presence of such a zone (whether from depth recording, neuroimaging, or clinical outcome of lobectomy) does not amount, as several authors have claimed, to validation of dipole modelling.

\section{RELATIONS BETWEEN ICTAL AND INTERICTAI} EVENTS

It is particularly important that surface interictal epileptiform activity can be driven by structures that do not trigger seizures. Moreover, in one out of five patients only those interictal deep spikes that were contralateral to the ictal onset zone could be detected on the surface. This obviously poses a difficult physiological problem to solve, regardless of discussions about the ideal mathematical model for the source, as not all structures that seem to be active interictally are able to trigger seizures. This can be a neurophysiological substrate to the common clinical finding that the analysis of interictal activity may be misleading in lateralising the seizure onset in partial epilepsy. ${ }^{33}$

Ictal electrical events in complex partial seizures can remain confined to a limited number of deep contacts for a few seconds before activity is recorded on the surface (fig 3 ). The propagation of ictal activity has been extensively studied since depth recordings became available. ${ }^{1}$ It has been reported that the spread of seizures of mesial temporal origin takes place along preferential anatomical pathways, most often to frontoorbital areas, posterior cingulate gyrus, and contralateral hippocampus. Apart from the last, these projections seem to largely coincide with those followed by interictal discharges. The reason why ictal activity can remain relatively confined for seconds whereas interictal activity can spread within milliseconds, causing a less sustained phenomenon and much less symptomatology, is not known but it poses an important fundamental question about the genesis and propagation of epileptiform activity.

\section{CLINICAL RELEVANCE}

This work corroborates the traditional interpretation of EEG as being generated by cellular activity taking place near the electrode that records discharges, and emphasises the importance of ictal recordings. Propagation of activity is likely, however, to follow certain neural pathways. For instance, most activity from deep sources seems to propagate to the ipsilateral neocortex. Therefore, information about deep epileptogenic foci could in principle be obtained from detailed mapping of their neocortical projection areas by means of scalp EEG or MEG. In this respect, the association of definite interictal patterns with surgical outcome would be of paramount clinical importance and the combined use of scalp EEG and MEG could prove very helpful in this task. ${ }^{34}$ As large areas of the neocortex can be simultaneously active during interictal events, the characterisation of electromagnetic sources as a convoluted strip of dipoles, perhaps superimposed on neocortical grey matter identified on radiological images, seems to be a more appropriate mathematical model than isolated dipoles or clusters of dipoles. Whether the topography of such neocortical dipole arrays is reliably predictive of the location of a deep pacemaker has yet to be established.

1 Wieser HG. Human limbic seizures: EEG studies, origin and patterns of spread. In: Meldrum BS, Ferrendelli JA, . $127-38$.

2 Spencer SS. Cortical and intercortical seizure spread. In Meldrum BS, Ferrendelli JA, Wieser HG, eds. Curren problems in epilepsy. Vol 6, Anatomy of epileptogenesis. London: John Libbey, 1988:139-54.

3 Ricci GB, Romani GL, Salustri C, Pizzella V, Torrioli G Buonomo, et al. Study of focal epilepsy by multichanne neuromagnetic measurements. Electroencephalogr Clin Neurophysiol 1987;66:358-68.

4 Rose DF, Sato S, Smith PD, Porter RJ, Theodore WH, Friauf W, et al. Localisation of magnetic interictal discharges in temporal lobe epilepsy. Ann Neurol 1987;22. 348-54.

5 Salustri C, Chapman RM. A simple method for 3dimensional localisation of epileptic activity recorded by Neurophysiol 1989;73:473-8. 
6 Stefan H, Schneider S, Abraham-Fuchs K, Pawlick G, Feistel H, Bauer J, et al. The neocortico to mesiobasal limbic propagation of focal epileptic activity during the
spike-wave complex. Electroencephalogr Clin Neurophysiol 1991;79:1-10.

7 Sutherling WW, Crandall PH, Cahan LD, Barth DS. The magnetic field of epileptic spikes agrees with intracranial magnetic field of epileptic spikes agrees with intracranial localisations

8 Barth DS, Baumgartner C, Sutherling WW. Neuromagnetic field modelling of multiple brain regions producing interictal spikes in human epilepsy. Electroencephalogr Clin Neurophysiol 1989;73:389-402.

9 Lutkenhoner B. Frequency domain localisation of intracerebral dipolar sources. Electroencephalogr Clin Neurophysiol 1992;82:112-8.

10 Ebersole JS. Equivalent dipole modelling-a new EEG method for localisation of epileptogenic foci. In Recent advances in epilepsy, Vol 5. London: Churchill Livingstone, 1992:51-71.

11 Sarvas J. Basic mathematical and electromagnetic concepts of the biomagnetic inverse problem. Phys Med Biol 1987; 32:11-22.

12 Hellstrand E, Abraham-Fuchs K, Schneider S. Magnetoencephalographic localisation of epileptic dipole activity along gyrus cinguli in patients with pharmacoresistant epilepsy. 8th gyrus cinguli in patients with pharmacoresistant epilepsy. 8th Germany, 1991 .

13 Cohen D, Cuffin N, Kazutomo Y, Maniewski R, Purcell C, Rees-Cosgrove G, et al. MEG versus EEG localisation tests using implanted sources in the human brain Ann Neurol 1990;28:811-7.

14 Mauguière F. A consensus statement on relative merits of EEG and MEG. Electroencephalogr Clin Neurophysiol 1992;82:317-9.

15 Binnie CD, Elwes RDC, Polkey CE, Volans A. Utility of stereoelectroencephalography in preoperative assessment of temporal lobe epilepsy. F Neurol Neurosurg Psychiatry 1994;57:58-65.

16 Wieser HG, Elger CE, Stodieck SRG. The 'Foramen Ovale Electrode': a new recording method for the preoperative evaluation of patients suffering from mesiobasal temporal lobe epilepsy. Electroencephalogr Clin Neurophysiol 1985;61:314-22.

17 Binnie CD, Dekker E, Smit A, et al. Practical considerations in the positioning of EEG electrodes. Electrotions in the positioning of EEG electrod
encephalogr Clin Neurophysiol 1982;53:453-8.

18 Van Veelen CWM, Debets RM, Van Huffelen AC, et al. Combined use of subdural and intracerebral electrodes in preoperative evaluation of epilepsy. Neurosurgery 1990;26:93-101.

19 Barber DC, Brown BH. Applied potential tomography. f Phys E: Sci Instrument 1984;17: 723-33.
20 Nicholson PW. Specific impedance of cerebral white matter. Exp Neurol 1965;13:386-401.

21 Kosterich JD, Foster KR, Pollack SR. (1983) Dielectric permittivity and electrical conductivity of fluid saturated bone. IEEE Trans Biomed Eng 1993;30:81-6.

22 Cobb W, Sears TA. (1960) Study of the transmission of potentials after hemispherectomy. Electroencephalogr Clin potentials after hemispherect 1960;12:371-83.

23 Cooper R, Winter AL, Crow HJ, Grey Walter W. Comparison of subcortical, cortical and scalp activity using chronically indwelling electrodes in man. Electrousing chronically indwelling electrodes in man.

24 Cuffin BN, Cohen D, Yunokuchi K, Maniewski R, Purcel C, Cosgrove GR, et al. Tests of EEG localisation accuracy using implanted sources in the human brain. Ann Neurol 1991;29:132-8.

25 Lopes da Silva FH, Witter MP, Boeijinga PH, Lohman AHM. Anatomic organisation and physiology of the limbic cortex. Physiol Rev 1990;70:453-511.

26 Pandya DN, Yeterian EH. Hodology of limbic and related structures: cortical and comissural connections. In Wieser HG, Elger CE, eds. Presurgical evaluation of epileptics. Berlin: Springer-Verlag, 3-14.

27 Amaral DG, Price JL. Amygdalo-cortical projections in the monkey (Macaca fascilularis). $\mathcal{F}$ Comp Neurol 1984;230: monkey

28 Hughes JR. A statistical analysis on the location of EEG abnormalities. Electroencephalogr Clin Neurophysiol 1960;

29 Truett A, McCarthy G, Wood CC, Darcey TM, Spencer DD, Williamson PD. Human cortical potentials evoked by stimulation of the median nerve. I. Cytoarchitectonic areas generating short-latency activity. $\mathcal{F}$ Neurophysiol 1989;62:694-710.

30 Patton HD. Special properties of nerve trunks and tracts. In: Ruch T, Patton HD, eds. Physiology and biophysics. Vol IV. Philadelphia: WB Saunders Co, 1982 101-27.

31 McBride MC, Binnie CD, Janota I, Polkey CR. Predictive value of intraoperative electrocorticograms in resective epilepsy surgery. Ann Neurol 1991;30:526-32.

32 Hosek RS, Sances A, Jodat RW, Larson SJ. The contributions of intracerebral currents to the EEG and evoked potentials. IEEE Trans Biomed Eng 1978;25: 405-13.

33 Engel J, Driver MV, Falconer MA. Electrophysiological correlates of pathology and surgical results in temporal

34 Guy CN, Alarcon G, Walker SR, Binnie CD, Smith S, Sveindjornsdottir S. Model calculations of the surface electromagnetic fields produced by extended current sources. application to epilepsy. COMAC conference on Epilepsy and Biomagnetism, Cambridge, UK 1991. 